\title{
ORIGINAL
}

\section{ANÁLISIS DE LA SUPERVIVENCIA EN PACIENTES CON CÁNCER MÚLTIPLE. ASTURIAS, 1975-2004 (*)}

\begin{abstract}
Alberto Lana Pérez (1), María Victoria Folgueras Sánchez (1, 2), Sandra Díaz Rodríguez (1), María del Olivo del Valle Gómez (1), Antonio Cueto Espinar (1), María Luisa López González (1)

(1) Departamento de Medicina. Área de Medicina Preventiva y Salud Pública. Instituto Universitario de Oncología del Principado de Asturias. Universidad de Oviedo.

(2) Servicio de Anatomía Patológica. Hospital Universitario Central de Asturias. Instituto Universitario de Oncología del Principado de Asturias.
\end{abstract}

\section{RESUMEN}

Fundamento. Casi uno de cada diez supervivientes de cáncer sufre un segundo primario. Algunos estudios intentan clarificar las causas pero escasean los que estudian la variable tiempo. Nuestro objetivo fue describir esta variable en pacientes con cáncer múltiple (CM) y detectar otras asociadas con el tiempo libre de CM y la supervivencia.

Métodos. Se diseñó un estudio descriptivo y se utilizaron el método de Kaplan Meyer y la Regresión de Cox para estudiar los pacientes con $\mathrm{CM}$ incluidos en el registro de tumores del Hospital Central de Asturias.

Resultados. El tiempo 'primer síntoma-diagnóstico' se redujo significativamente del primer al segundo cáncer (de 113 a 88 días). El tiempo entre los dos primeros cánceres (mediana $=2,93$ años) fue menor en las personas de más edad $(R R=1,03)$, con cánceres primarios avanzados $(R R=1,55)$, tratados con cirugía paliativa $(R R=2,67)$ o quimioterapia $(\mathrm{RR}=1,51)$ y con un segundo cáncer localizado en la próstata $(\mathrm{RR}=1,67)$. El $60,9 \%$ sobrevivió más de 5 años desde el primer diagnóstico y el 19,1\% desde el segundo. Estas probabilidades disminuyeron con la edad $(\mathrm{RR}=1,04)$ y estadios avanzados $(\mathrm{RR}=2,48)$, sin embargo, localizaciones con buen pronóstico (próstata, mama y piel) y casi cualquier tratamiento quirúrgico o radiológico aumentan la supervivencia.

Conclusión. Los médicos deben estar alerta ante nuevos síntomas de cáncer en pacientes con neoplasias previas durante su periodo de seguimiento para mejorar el diagnóstico precoz del CM. La supervivencia en estos pacientes depende en gran medida de la localización y estadio del cáncer implicado, pero también del tipo de tratamiento.

Palabras clave: Neoplasia múltiple. Supervivencia. Modelo de Riesgos Proporcionales. Pronóstico. Prevención \& control.

Correspondencia:

María Luisa López González

Facultad de Medicina

C/ Julián Clavería s/n. 33006 Oviedo

Correo electrónico: lopez@uniovi.es.

\section{ABSTRACT}

\section{Survival Analysis in Multiple Cancer Patients in Asturias, Spain, 1975-2004}

Background. Almost one in ten survivors of cancer suffers a second primary. Some studies try to clarify the causes, but there are few that study the time variable. Our research aim was to describe this variable in multiple cancer (MC) patients and to detect others associated with time without MC and survival.

Methods. We performed a descriptive study, and the Kaplan Meyer method and Cox Regression were used to study the patients with MC included in the tumour registry of the reference hospital of Asturias.

Results. Time between the first symptom and diagnosis was significantly reduced in the second tumour when compared with the first one (113 to 88 days). Time between the first two tumours (median=2.93 years) and the risk of an earlier second primary was higher in older people $(\mathrm{RR}=1,03)$ with: primaries diagnosed in advanced stage $(R R=1.55)$, treated with palliative surgery $(R R=2.67)$ or chemotherapy $(R R=1.51)$; second neoplasm located in the prostate $(\mathrm{RR}=1.67) .60 .9 \%$ survived at least 5 years after the first diagnosis and $19.1 \%$ after the second. These probabilities decreased with age $(R R=1.04)$ and advanced stages $(R R=2.48)$. Nevertheless, locations with good prognosis (prostate, breast or skin) and almost any treatment with surgery or radiotherapy increase survival.

Conclusion. Physicians should be alert to new cancer symptoms in patients with previous neoplasms during the followup period to improve early MC diagnosis. Survival in MC patients is acceptable, and it largely depends on the location and stage of the cancer involved, but also upon the strategy of treatment.

Key words: Neoplasms, Second Primary. Survival Analysis. Proportional Hazards Models. Time factors. Prognosis. Prevention and control.

(*) Fondo de Investigación Sanitaria (PI 03/0542) 


\section{INTRODUCCIÓN}

El cáncer múltiple $(\mathrm{CM})$ es definido por la International Agency for Research on Cancer (IARC) ${ }^{1}$ como "la existencia de dos o más cánceres primarios que no son ni extensión, ni recurrencia, ni metástasis y que están localizados en lugares diferentes, o en el mismo lugar si son histológicamente diferentes". Aunque no es un fenómeno nuevo su incidencia en las últimas décadas está aumentando en casi todo el mundo desarrollado ${ }^{2,3}$. El porcentaje de CM sobre el total de cáncer es muy variable, pero supera el 10\% en algunas poblaciones ${ }^{4}$. En Asturias este porcentaje casi se ha cuadruplicado en los últimos 25 años y ya alcanza el $9,21 \%{ }^{5}$, aunque todavía es menor que en EEUU ${ }^{6}$ donde llega al $16 \%$. Esta tendencia puede estar relacionada con el mayor éxito de las terapias para alcanzar remisiones a largo plazo $^{7} \mathrm{o}$ con el potencial efecto carcinogénico del tratamiento del primer cáncer ${ }^{8}$, pero también con la persistencia de conductas de riesgo de cáncer tras el diagnóstico del primer tumor.

El aumento de la incidencia de CM debería promover un mayor interés en la investigación epidemiológica de esta patología. Hay bastantes estudios descriptivos y analíticos que tratan de clarificar la etiología ${ }^{3,7-10}$, posiblemente compartida por el primer y subsiguientes cánceres, y que buscan asociaciones entre distintas localizaciones primarias en un intento de obtener claves para la prevención. Pero escasean los estudios centrados en la variable epidemiológica tiempo y los pocos que hay no son determinantes $^{11-14}$.

Sin embargo, el estudio de al menos tres variables de tiempo puede resultar interesante: el tiempo transcurrido entre el primer síntoma percibido por el paciente relacionado con el cáncer y su diagnóstico definitivo; el transcurrido entre el diagnóstico de un cáncer y el siguiente; $\mathrm{y}$, finalmente, el tiempo de supervivencia a partir de cada diagnóstico en función del número de cánceres primarios padecidos. El primero de estos tiempos puede servir como indicador indirecto del nivel de educación sanitaria del paciente y del celo de los profesionales sanitarios en la aplicación de medidas de prevención. Los otros periodos podrían aportar información útil para saber si existe oportunidad de prevención del CM y para conocer el perfil de paciente que más se beneficiaría de los potenciales programas preventivos.

Los registros de tumores (RT) tienen algunas limitaciones ligadas a su nivel de calidad $^{15,16}$, pero han sido utilizados en muchos países con fines descriptivos y analíticos ${ }^{2-3,7-10}$ porque también son un gran recurso para estudiar CM. Según AntonCulver ${ }^{17}$ la prevención del cáncer debería ser la primera meta de los RT y, en este sentido, los datos de tiempo incluidos en los RT pueden aportar claves para la toma de decisiones tanto terapéuticas como, sobre todo, preventivas $^{18}$.

El objetivo principal de este trabajo fue describir la variable tiempo en pacientes con $\mathrm{CM}$ inscritos en un RT hospitalario mediante el análisis del tiempo 'síntomadiagnóstico', el tiempo entre los distintas neoplasias primarios y el tiempo de supervivencia. Como objetivo secundario se planteó la búsqueda de variables asociadas con el padecimiento precoz de CM y con una menor supervivencia.

\section{SUJETOS Y MÉTODOS}

Fuente de datos y materiales: Estudio descriptivo de la cohorte retrospectiva de pacientes con CM incluidos en el RT del Hospital Universitario Central de Asturias desde la fecha de su creación. Como criterio de inclusión se utilizó el haber sido 
diagnosticado de CM entre 1975 y 2004 según los requisitos de la IARC ${ }^{1}$, es decir, haber padecido durante este periodo al menos un cáncer que no fuera el primero, ni tampoco metástasis o recurrencia de uno anterior.

La fuente de datos primaria fue el RT del Hospital Universitario Central de Asturias, que es el hospital de referencia de la Comunidad Autónoma y el único que disponía de servicio de radioterapia a la conclusión del estudio. Este registro hospitalario fue uno de los primeros en implantarse en España y colabora con la IARC desde hace dos décadas a través del RT poblacional.

La información de la muestra $(\mathrm{N}=2.737)$ fue manejada anónimamente, con el permiso del Comité de Ética del Hospital, e introducida en una base de datos en SPSS 15.0 (SPSS Inc, Chicago, Illinois USA) que recogía, entre otras variables, características del: a) Paciente: edad (en años) al diagnóstico de cada neoplasia, sexo y área sanitaria en la que residía el paciente, según las ocho existentes en la comunidad; b) Cáncer: localización (por órganos afectados o agrupados por sistemas), tipo histológico, estadio y tratamiento de cada cáncer; c) Tiempo: tiempo 'primer síntoma-diagnóstico' expresado en días, tiempo entre los distintos tumores primarios y tiempo de supervivencia expresados en años. Localización, histología y estadio fueron codificados siguiendo la tercera edición de la Clasificación Internacional de Enfermedades para Oncología ${ }^{19}$, las International rules for multiple primary cancers $^{1}$ y los grupos histológicos propuestos por la IARC ${ }^{20}$. Para simplificar algunos análisis, las categorías originales fueron agrupadas conforme se recoge en la tabla 1 de resultados.

Los análisis de supervivencia fueron realizados sólo con aquellos pacientes cuyo estado vital pudo ser confirmado $(n=1.774)$, porque en esta submuestra la fiabilidad de los datos referidos a la mortalidad (estado vital y causa de muerte) pudo ser comprobada en las historias clínicas, en los registros de defunción del Instituto Nacional de Estadística y en las bases de datos de mortalidad oficiales de la Consejería de Sanidad.

Análisis de datos: Se realizó estadística descriptiva y se calcularon medias e intervalos de confianza (IC95\%) de los tiempos estudiados, así como las respectivas medianas y los rangos intercuartílicos (RIC). Aplicamos el test de Kolmogorov-Smirnov para evaluar si las variables cuantitativas cumplían los criterios de normalidad y, en función del resultado, utilizamos el test de Wilcoxon (muestras relacionadas) para detectar diferencias significativas entre los distintos periodos de tiempo, según el número de cánceres sufridos.

Con el método de Kaplan-Meier se estimó la función del tiempo transcurrido hasta la aparición del cáncer múltiple y la de la supervivencia. Las curvas de supervivencia fueron comparadas mediante Log-rank test.

Se realizaron varios análisis multivariantes usando el Modelo de Riesgo Proporcional de Cox (método Introducir en 3 pasos) para estimar el riesgo relativo de padecer CM precoz y de morir por cáncer. En general, estos análisis se ajustaron con todas las variables de persona (paso 1), cáncer (paso 2) y tiempo (paso 3 ) incluidas en la tabla 1 de resultados para valorar su posible efecto pronóstico, aunque previamente se habían realizado varios análisis bivariantes para la selección de covariables y decidir el tipo de codificación más adecuado. El modelo final multivariante llevado a cabo para determinar las variables asociadas con el padecimiento de un $\mathrm{CM}$ precoz incluyó todas las variables relativas al primer cáncer y aquellas referidas al segundo cáncer que tenían que ver con el retraso diagnóstico, localización, estadio e histología. Para el estudio de la supervivencia se construyó un modelo final que tenía como variable dependiente el tiempo desde el segundo diagnóstico 
hasta la finalización del periodo de seguimiento (supervivencia después del diagnóstico del CM) y se ajustó con las variables de la tabla 1 referidas a los dos primeros tumores (excepto "Lugar de residencia", ya que no se relacionaba de forma significativa y no modificaba el resto de RR). En este análisis no se incluyeron los datos relativos al tercer cáncer para no limitar la información disponible. Se consideraron significativos aquellos valores de $\mathrm{p}<0,05$.

\section{RESULTADOS}

Se incluyó en el estudio a los 2.737 pacientes con CM $(67,2 \%$ hombres; $p<0,001$ ), que padecieron 5.629 cánceres en total: 2.591 con únicamente 2 cánceres primarios, 137 con 3 y 9 con 4 . La tabla 1 resume las principales características personales y aquéllas relacionadas con los tumores de los pacientes que padecieron hasta 3 cánceres. Asimismo, muestra el tiempo transcurrido entre el primer síntoma y el diagnóstico definitivo. Dicho tiempo se redujo significativamente $(p<0,001)$ entre el primer cáncer (mediana $=113$ días) y el segundo (mediana=88), pero volvió a aumentar significativamente $(p<0,001)$ con ocasión del tercero (mediana=105). No se analizó el tiempo medio del cuarto cáncer debido al reducido número de casos.

El estudio del tiempo transcurrido entre los tres primarios arrojó los siguientes valores medios: 4,88 años entre el $1^{\circ} \mathrm{y}$ el $2^{\circ}$ (IC95\%: 4,67-5,09) y 3,03 años entre el $2^{\circ}$ y el $3^{\circ}$ (IC95\%: 2,40-3,65). La mediana de dichos intervalos fue 2,93 (RIC: 0,77,05) y 1,52 (RIC: 0,37-4,58) respectivamente; y la diferencia entre ambos significativa $(p<0,001)$.

El tiempo medio transcurrido sin cáncer múltiple fue el que medió entre el diagnóstico definitivo del primero y del segundo cáncer, esto es los 4,88 años citados en el
Tabla 1

Resumen de las principales variables incluidas en el estudio

\begin{tabular}{|c|c|c|c|}
\hline Variables & $\begin{array}{c}\text { Primer } \\
\text { cáncer } \\
\mathbf{N}=2.737\end{array}$ & $\begin{array}{l}\text { Segundo } \\
\text { cáncer } \\
\mathrm{N}=\mathbf{2 . 7 3 7}\end{array}$ & $\begin{array}{l}\text { Tercer } \\
\text { cáncer } \\
\mathrm{N}=137\end{array}$ \\
\hline $\begin{array}{l}\text { Pacientes } \\
\text { Edad }[\text { Mediana (RIC)] } \\
\text { Sexo }[\mathrm{N}(\%)] \\
\text { Lugar de residencia }[\mathrm{N}(\%)] 1.26\end{array}$ & \multicolumn{3}{|c|}{$\begin{array}{c}64(55-71) \quad 69(61-73) \quad 70(64-75,2) \\
1.840 \text { hombres }(67,2 \%) \\
\text { \%)] } 1.262 \text { en área de referencia }(46,1 \%)\end{array}$} \\
\hline $\begin{array}{l}\text { Cáncer } \\
\text { Localización [\%] } \\
\text { Piel no melanoma } \\
\text { Laringe } \\
\text { Mama } \\
\text { Vejiga } \\
\text { Próstata } \\
\text { Tráquea - Pulmón } \\
\text { Colon } \\
\text { Resto }\end{array}$ & $\begin{array}{l}15,7 \\
9,9 \\
8,6 \\
8,5 \\
6,1 \\
5,8 \\
5,0 \\
40,4\end{array}$ & $\begin{array}{l}13,8 \\
2,9 \\
5,2 \\
6,1 \\
5,8 \\
18,6 \\
5,9 \\
41,7\end{array}$ & $\begin{array}{l}14,4 \\
1,4 \\
2,1 \\
11,6 \\
5,5 \\
13,7 \\
6,2 \\
45,1\end{array}$ \\
\hline $\begin{array}{l}\text { Estadío [\%] } \\
\text { Desconocido } \\
\text { Local } \\
\text { Regional } \\
\text { Avanzado } \\
\text { No aplicable }\end{array}$ & $\begin{array}{l}20,3 \\
50,6 \\
13,9 \\
7,0 \\
8,2\end{array}$ & $\begin{array}{l}11,4 \\
46,8 \\
17,3 \\
14,8 \\
9,7\end{array}$ & $\begin{array}{l}13,7 \\
45,9 \\
13,7 \\
13,7 \\
13,0\end{array}$ \\
\hline $\begin{array}{l}\text { Histología [\%] } \\
\text { Carcinomas epidermoides } \\
\text { Adenocarcinomas } \\
\text { Otros carcinomas específicos } \\
\text { Carcinomas inespecíficos } \\
\text { Linfomas } \\
\text { Sarcomas y tejidos blandos } \\
\text { Leucemias } \\
\text { Otros tipos específicos de tumor } \\
\text { Tumores inespecíficos }\end{array}$ & $\begin{array}{l}49,7 \\
31,1 \\
2,3 \\
3,9 \\
4,3 \\
1,2 \\
2,6 \\
4,1 \\
0,9\end{array}$ & $\begin{array}{l}42,7 \\
35,3 \\
5,9 \\
4,4 \\
2,5 \\
1,7 \\
2,1 \\
3,7 \\
1,6\end{array}$ & $\begin{array}{l}46,2 \\
29,7 \\
4,8 \\
2,8 \\
1,4 \\
2,8 \\
1,4 \\
8,3 \\
2,8\end{array}$ \\
\hline $\begin{array}{l}\text { Tratamiento } \\
\text { Cirugía [\%] } \\
\text { No } \\
\text { Sí, radical } \\
\text { Sí, paliativa }\end{array}$ & $\begin{array}{l}29,0 \\
69,8 \\
1,2\end{array}$ & $\begin{array}{l}42,3 \\
55,0 \\
2,7\end{array}$ & $\begin{array}{l}39,7 \\
56,9 \\
3,4\end{array}$ \\
\hline $\begin{array}{l}\text { Radioterapia [\%] } \\
\text { No } \\
\text { Sí, radical } \\
\text { Sí, paliativa } \\
\text { Complementaria }\end{array}$ & $\begin{array}{l}64,9 \\
18,4 \\
1,1 \\
15,6\end{array}$ & $\begin{array}{l}72,6 \\
14,1 \\
4,0 \\
9,3\end{array}$ & $\begin{array}{l}76,7 \\
12,4 \\
4,1 \\
6,8\end{array}$ \\
\hline $\begin{array}{l}\text { Quimioterapia [\%] } \\
\text { No } \\
\text { Sí, radical } \\
\text { Sí, paliativa }\end{array}$ & $\begin{array}{l}87,3 \\
12,4 \\
0,3\end{array}$ & $\begin{array}{l}82,3 \\
16,5 \\
1,2\end{array}$ & $\begin{array}{l}85,6 \\
13,0 \\
1,4\end{array}$ \\
\hline $\begin{array}{l}\text { Tiempo } \\
\text { Días entre el primer síntoma y } \\
\text { el diagnóstico. [Mediana (RIC)] }\end{array}$ & $\begin{array}{c}113 \\
(54-309,7)\end{array}$ & $\begin{array}{c}88 \\
(36-188)(\end{array}$ & $\begin{array}{c}105 \\
(9,5-242,5)\end{array}$ \\
\hline $\begin{array}{l}\text { Supervivencia [\%] } \\
\text { A los } 5 \text { años } \\
\mathrm{A} \operatorname{los} 10 \text { años }\end{array}$ & $\begin{array}{l}60,8 \\
30,8\end{array}$ & $\begin{array}{c}19,1 \\
5,4\end{array}$ & $\begin{array}{l}9,5 \\
3,8\end{array}$ \\
\hline
\end{tabular}

párrafo anterior (mediana $=2,93$; RIC: 0,77,05). La figura 1 muestra la función del tiempo desde el diagnóstico del primer primario hasta la aparición de cáncer múltiple en los 2.737 pacientes. En el 12,5\% de los casos el CM apareció durante el mes que sigue al primer diagnóstico y en el $29,3 \%$ en el transcurso del primer año. 
Figura 1

Función del tiempo libre de cáncer múltiple en años

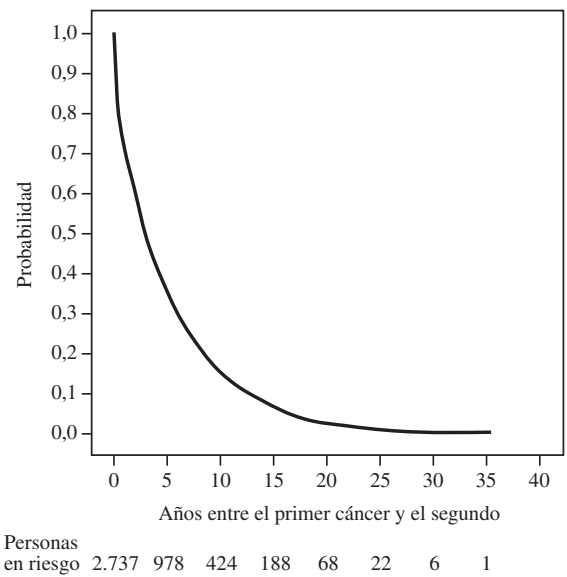

El análisis multivariante del tiempo libre de CM reveló que dicho tiempo se asocia significativamente con las variables incluidas en la tabla 2. Las personas de mayor edad (RR: 1,03) con un primer cáncer diagnosticado en estadio avanzado (RR: 1,55) y que fueron sometidas a cirugía paliativa (RR: 2,67) o tratadas con quimioterapia radical (RR: 1,51 ) durante el primer cáncer, padecieron un segundo primario precozmente, sobre todo si este último se localizó en la próstata (RR: 1,67). Por el contrario, sufrir un cáncer de mama como primera manifestación cancerosa disminuyó este riesgo (RR: 0,47).
El 60,9\% de los pacientes $(n=1.774)$ tuvo una supervivencia superior a los 5 años desde el diagnóstico del primer cáncer (figura 2a), aunque resultó variable en función de su localización: entre el 41,9\% del cáncer de próstata y el $79,4 \%$ del de mama, como extremos de supervivencia a los 5 años de los cánceres más comunes. A los 10 años el porcentaje de supervivencia tras el primer cáncer fue del $30,8 \%$. Los tiempos de supervivencia medios de los pacientes fueron: 7,21 años (IC95\%: 6,91-7,51) en los 1.669 pacientes con 2 neoplasias; 10,19 (IC95\%: 8,84-11,55) en los 99 pacientes con 3 cánceres y 18,80 (IC95\%: 7,74-29,86) en los 6 pacientes que lograron acumular 4 primarios. Las respectivas medianas fueron: 6,38 (RIC: 3,07-11,33); 8,84 (RIC: 5,24-14,32) y 16,46 (RIC: 10,8-26,73). En todos los casos las diferencias entre estos tiempos fueron significativas $(p<0,001)$. Desde el diagnóstico de $\mathrm{CM}$, es decir, del segundo cáncer primario, el porcentaje de supervivencia media a los 5 años fue del 19,1\% (figura 2b) y los tiempos medios de supervivencia en los mismos pacientes fueron, respectivamente: 1,99 (IC95\%: 1,86-2,13); 4,94 (IC95\%: 3,965,93) y 7,17 (IC95\%: 4,35-10,01) años. También en este caso la mediana de años de supervivencia tras el segundo primario se incrementó significativamente $(p<0,001)$ en función del número de cánceres sufridos: 1,59 (RIC: 0,56-3,92); 4,11 (RIC: 1,64-6,72) y 6,74 (RIC: 4,68-9,93) años.

Tabla 2

Análisis multivariante del tiempo libre de cáncer múltiple (tiempo entre el primer y segundo tumor)

\begin{tabular}{|c|c|c|c|c|}
\hline & \multirow{2}{*}{$\mathbf{R R}$} & \multicolumn{2}{|c|}{ IC 95\% para RR } & \multirow{2}{*}{$p$} \\
\hline & & Inferior & Superior & \\
\hline Edad al diagnóstico I & 1,03 & 1,02 & 1,04 & $<0,001$ \\
\hline $\begin{array}{l}\text { Estadio local } \\
\text { Estadio avanzado I }\end{array}$ & $\begin{array}{c}1^{\mathrm{a}} \\
1,55\end{array}$ & 1,02 & 2,35 & 0,038 \\
\hline $\begin{array}{l}\text { Colon } \\
\text { Mama I } \\
\text { Próstata II }\end{array}$ & $\begin{array}{c}1^{\mathrm{a}} \\
0,47 \\
1,67\end{array}$ & $\begin{array}{l}0,29 \\
1,02\end{array}$ & $\begin{array}{l}0,76 \\
2,69\end{array}$ & $\begin{array}{l}0,002 \\
0,042\end{array}$ \\
\hline $\begin{array}{l}\text { No realizada Cirugía } \\
\text { Cirugía paliativa I }\end{array}$ & $\begin{array}{c}1^{\mathrm{a}} \\
2,67\end{array}$ & 1,18 & 6,05 & 0,019 \\
\hline $\begin{array}{l}\text { No administrada quimioterapia } \\
\text { Quimioterapia radical I }\end{array}$ & $\begin{array}{c}1^{\mathrm{a}} \\
1,51\end{array}$ & 1,11 & 2,05 & 0,008 \\
\hline
\end{tabular}

$1^{\mathrm{a}}$ = Categoría de referencia

$\mathrm{I}$ = Variables referidas al primer tumor; $\mathrm{II}=$ Variables referidas al segundo tumor 
Figura 2

Función de supervivencia según el número de tumores


El análisis multivariante de la supervivencia $(n=1.774$, censurado: $27,6 \%)$ desde el diagnóstico del CM mostró las asocia-

ciones significativas que se muestran en la tabla 3. Tuvieron una supervivencia menor aquellos pacientes con cánceres primarios

Tabla 3

Análisis multivariante de la superficie a partir del diagnóstico del CM

\begin{tabular}{|c|c|c|c|c|}
\hline & \multirow{2}{*}{$\mathbf{R R}$} & \multicolumn{2}{|c|}{ IC 95\% para RR } & \multirow{2}{*}{$p$} \\
\hline & & Inferior & Superior & \\
\hline Mujer & 0,70 & 0,59 & 0,84 & $<0,001$ \\
\hline Edad al diagnóstico II & 1,04 & 1,01 & 1,08 & 0,025 \\
\hline $\begin{array}{l}\text { Colon } \\
\text { Próstata I } \\
\text { Otros piel II } \\
\text { Mama II } \\
\text { Próstata II }\end{array}$ & $\begin{array}{c}1^{\mathrm{a}} \\
0,67 \\
0,53 \\
0,55 \\
0,35\end{array}$ & $\begin{array}{l}0,54 \\
0,38 \\
0,36 \\
0,25\end{array}$ & $\begin{array}{l}0,83 \\
0,74 \\
0,84 \\
0,49\end{array}$ & $\begin{array}{c}<0,001 \\
<0,001 \\
0,006 \\
<0,001\end{array}$ \\
\hline $\begin{array}{l}\text { Carcinomas epidermoides } \\
\text { Adenocarcinomas I } \\
\text { Linfomas I } \\
\text { Linfomas II } \\
\text { Leucemias II }\end{array}$ & $\begin{array}{c}1^{\mathrm{a}} \\
0,78 \\
0,68 \\
0,42 \\
0,42\end{array}$ & $\begin{array}{l}0,63 \\
0,47 \\
0,28 \\
0,27\end{array}$ & $\begin{array}{l}0,97 \\
0,98 \\
0,62 \\
0,65\end{array}$ & $\begin{array}{l}0,029 \\
0,039 \\
<0,001 \\
<0,001\end{array}$ \\
\hline $\begin{array}{l}\text { Estadio local } \\
\text { Regional I } \\
\text { Regional II } \\
\text { Avanzado II }\end{array}$ & $\begin{array}{c}1^{\mathrm{a}} \\
1,21 \\
1,71 \\
2,48\end{array}$ & $\begin{array}{l}1,01 \\
1,46 \\
2,04\end{array}$ & $\begin{array}{l}1,46 \\
2,01 \\
3,02\end{array}$ & $\begin{array}{c}0,04 \\
<0,001 \\
<0,001\end{array}$ \\
\hline $\begin{array}{l}\text { No realizada Cirugía } \\
\text { Cirugía radical I } \\
\text { Cirugía paliativa I } \\
\text { Cirugía radical II }\end{array}$ & $\begin{array}{c}1^{\mathrm{a}} \\
0,72 \\
0,52 \\
0,46\end{array}$ & $\begin{array}{l}0,59 \\
0,31 \\
0,39\end{array}$ & $\begin{array}{l}0,89 \\
0,86 \\
0,55\end{array}$ & $\begin{array}{c}0,002 \\
0,012 \\
<0,001\end{array}$ \\
\hline $\begin{array}{l}\text { No administrada Radioterapia } \\
\text { Radioterapia radical I } \\
\text { Radioterapia radical II } \\
\text { Radioterapia complementaria II }\end{array}$ & $\begin{array}{c}1^{\mathrm{a}} \\
0,65 \\
0,49 \\
0,67\end{array}$ & $\begin{array}{l}0,52 \\
0,39 \\
0,53\end{array}$ & $\begin{array}{l}0,82 \\
0,61 \\
0,85\end{array}$ & $\begin{array}{c}<0,001 \\
<0,001 \\
0,001\end{array}$ \\
\hline
\end{tabular}

$1^{\mathrm{a}}=$ Categoría de referencia

$\mathrm{I}$ = Variables referidas al primer tumor; $\mathrm{II}=$ Variables referidas al segundo tumor 
o secundarios diagnosticados en estadio no local, sobre todo si el segundo cáncer primario se diagnosticó en estadio avanzado (RR: 2,48), y las personas con mayor edad al diagnóstico de este segundo cáncer (RR: 1,04). Por otro lado, la probabilidad de sobrevivir aumentó en el caso de las mujeres (RR: 0,7) pero también si el primero de los cánceres asentó en la próstata (RR: 0,67 ) o bien el segundo en la piel (no melanoma) (RR: 0,53) en la mama (RR: 0,55$)$ o en la próstata (RR: 0,35). En nuestra serie también mejoró la supervivencia que el primer cáncer fuera un adenocarcinoma (RR: 0,78) o un linfoma (RR: 0,68) y que el segundo cáncer fuera un linfoma (RR: 0,42 ) o una leucemia (RR: 0,42). Recibir tratamiento quirúrgico o radiológico radical para alguno de los dos primarios se comportó como factor protector, aumentando el tiempo de supervivencia, así como la cirugía con intención paliativa del primer cáncer (RR: 0,52) o el tratamiento complementario con radioterapia del segundo (RR: 0,67).

\section{DISCUSIÓN}

Los tiempos transcurridos entre el primer síntoma y el diagnóstico definitivo son indeseablemente elevados, con medianas que oscilan entre 3 y 4 meses pero que se mueven en un rango que en ocasiones alcanza hasta los 10 meses de retraso diagnóstico. El tiempo del segundo cáncer primario es significativamente más corto que el de los otros dos, quizá porque padecer el primero sirve de aprendizaje para suscitar la alerta ante el segundo, pero no impide que se diagnostiquen en un estadio más avanzado. Además, el convencimiento de que ya se ha satisfecho el tributo al cáncer después de dos neoplasias y, afortunadamente, el reducido número de personas con tres procesos cancerosos, acaso hacen que en el tercero, si se produce, de nuevo se incremente el tiempo 'síntoma-diagnóstico', por lo que tiene de imprevisto para el paciente y los profesionales que le atienden. El diagnóstico precoz no parece habitual en nuestra serie, acaso por una escasa difusión de los consejos de prevención secundaria del Código Europeo contra el Cáncer y de los signos de alerta que incluye ${ }^{21}$, aun cuando está demostrado que la precocidad diagnóstica tiene un impacto en la supervivencia mayor que otras variables ${ }^{22}$. Este hecho es especialmente preocupante cuando se refiere a los segundos y terceros tumores primarios que en su mayoría se diagnostican durante el tiempo de seguimiento del cáncer previo.

La mediana del tiempo transcurrido entre los distintos tumores primarios es siempre inferior a cinco años, que suele ser el periodo mínimo de seguimiento clínico de cualquier tumor maligno, seguimiento que debería facilitar el diagnóstico precoz del CM si los profesionales estuvieran suficientemente alertas. En este sentido, Artac et $a l^{23}$ en un estudio similar, encontró una mediana de tiempo entre el primer y segundo cáncer análoga a la nuestra, en torno a los tres años, y recomienda seguimientos duraderos tras el tratamiento de los cánceres más comunes. Por tanto, los médicos deberían estar atentos a nuevos síntomas, no imputar cualquiera de ellos al proceso ya diagnosticado y sospechar la posible existencia de CM. Con los datos actuales, el cálculo de probabilidades establece que al menos uno de cada diez pacientes puede desarrollar CM. Por otro lado, que los tiempos entre los sucesivos primarios se vaya reduciendo puede ser debido al efecto deletéreo de los tumores malignos y tratamientos previos, o al natural proceso de envejecimiento del paciente.

La función del tiempo libre de CM muestra que éste puede aparecer precozmente, en el mismo año en que se diagnostica el primer cáncer, y hasta treinta años después. Pero, como encuentran Dong y Hemminki ${ }^{8}$ en un estudio con más de 600.000 pacientes, el periodo de máximo 
riesgo es la primera década, en la cual deberían extremarse las actividades de prevención de $\mathrm{CM}$, tanto primaria como secundaria. Estas actividades son de obligada aplicación a los pacientes con mayor probabilidad de CM precoz, los cuales, según nuestra investigación, son los de más edad y quienes recibieron quimioterapia radical o cirugía con intención paliativa para tratar un primer cáncer primario diagnosticado en estadío avanzado. Padecer un cáncer de mama disminuye el riesgo de un CM precoz, quizá porque los segundos primarios en personas con este tipo de neoplasias se asocian con el efecto carcinogénico que tienen los tratamientos a largo plazo ${ }^{24}$. Por otro lado, padecer un cáncer de próstata como segunda manifestación cancerosa se relaciona con un menor tiempo entre el primer y el segundo cáncer, aunque es posible que este hecho se deba a que en nuestro medio el screening oportunista del cáncer de próstata es muy frecuente ${ }^{25}$.

El estudio de la supervivencia para todos los tipos de cáncer de forma combinada puede ser un buen indicador de la atención sanitaria a las personas con cáncer que se presta en una población ${ }^{26}$. En nuestra serie los tiempos de supervivencia, una vez diagnosticado el primer cáncer, incluso el segundo, lógicamente crecen de manera significativa con el número de primarios, ya que sólo los supervivientes pueden padecer CM. Pero lo que interesa resaltar es que los tiempos de supervivencia oscilan entre los 6 y los 17 años tras el primer cáncer, y entre 1 y 7 años tras el diagnóstico de CM. Estas cifras, aunque inferiores a los 22,3 años de supervivencia tras el primer cáncer publicados por Artac et al ${ }^{23}$ son estimables. Es más, en torno a un $60 \%$ de los pacientes sobrevive al menos 5 años y alrededor de un $30 \%$ más de 10 años tras el primer diagnóstico, por lo que el adecuado tratamiento de éste debería incluir intervenciones preventivas rutinarias para la eliminación de factores de riesgo de cáncer y para el diagnóstico precoz de potenciales cánceres primarios subsiguientes. Recientemente el Instituto Nacional del Cáncer de EE.UU. publicó tasas de supervivencia inferiores a las de nuestra serie y, aún así, advertía de la creciente necesidad de tomar en consideración el $\mathrm{CM}^{10}$. Prestigiosos organismos sostienen que la prevención debe realizarse desde una perspectiva holística ${ }^{6}$ pero con especial esfuerzo en la mejora de los estilos de vida $^{27}$. En este sentido, hay evidencia científica de que factores de riesgo ligados a la alimentación, el tabaco y el sol persisten después del padecimiento de un cáncer, y de que no siempre se aplica la medicina curativa y la preventiva con el mismo celo $^{28}$, quizá porque esta última no es percibida como una prioridad por los profesionales sanitarios ${ }^{29}$.

El análisis crudo de la supervivencia mediante el Modelo de Riesgo Proporcional de Cox no está exento de limitaciones, pero parece igual de preciso que algunos análisis relativos si se tiene en cuenta la causa de la muerte ${ }^{30}$. En nuestro estudio, el análisis multivariante realizado para clarificar factores asociados a la supervivencia tras el CM muestra que tienen significativamente reducida la probabilidad de morir, por tanto, incrementada la supervivencia, los pacientes más jóvenes, más si son mujeres, diagnosticados en estadios locales y que recibieron cirugía o radioterapia radical como parte del tratamiento de alguno de sus cánceres (sobre todo radioterapia radical, ya que parece tener un efecto beneficioso al eliminar neoplasias simultáneas preco$\left.\operatorname{ces}^{31}\right)$. De ahí que deba extremarse la prevención en estas personas, sobre todo si cualquiera de los cánceres asienta en localizaciones que ofrecen buen pronóstico en la actualidad, como próstata, mama o piel no melanoma. Los principales tipos histológicos (adenocarcinomas, linfomas y leucemias) aumentaron la supervivencia significativamente, quizá porque la categoría utilizada como referencia (carcinomas epidermoides) se relaciona frecuentemente con cánceres del sistema respiratorio u 
órganos de cabeza y cuello, que tienen un mal pronóstico. Una vez más, un enfoque dirigido al abandono del hábito tabáquico, ya que es el principal factor de riesgo que estas últimas localizaciones comparten, podría ser beneficioso.

Nuestros resultados están limitados por la calidad de los datos del RT, como todos los estudios realizados a partir de tales registros. Aunque para el análisis de los diversos tiempos se han utilizado todos los casos incluidos en el registro durante el periodo de estudio, para el análisis de supervivencia sólo se han tenido en cuenta los casos en los que se pudo constatar fehacientemente la condición de vivo o muerto por cáncer, cruzando los datos del RT con las bases oficiales de mortalidad por cáncer de nuestra comunidad autónoma. Es por ello que las conclusiones sobre supervivencia sólo son aplicables a la década 19942004. Dicha supervivencia probablemente mejore la de las décadas anteriores, puesto que los modernos tratamientos consiguen remisiones cada vez más prolongadas ${ }^{32}$.

Los resultados del análisis del tiempo transcurrido entre el primer síntoma de cada proceso canceroso y el diagnóstico definitivo pueden estar afectados por un sesgo de memoria en lo que concierne a la primera de estas fechas, puesto que es autoinformada por el paciente, y por la falta de la información en algunas historias clínicas, sobre todo el referido al primer cáncer. Por otro lado, el recuerdo del primer síntoma podría tener distinta fiabilidad, según se refiera al primer cáncer o a los sucesivos. La convivencia con la enfermedad cancerosa genera cierto aprendizaje en el paciente respecto a su enfermedad, capaz de modificar los niveles de alerta frente al cáncer ${ }^{33}$. Los resultados referidos a terceros y cuartos primarios son provisionales, dado el escaso número de casos que integran el estudio.

Se requieren nuevas investigaciones a partir de registros fiables, procedentes de otras comunidades autónomas y del registro nacional de tumores, para clarificar y comparar la incidencia y supervivencia de CM, dentro y fuera de nuestras fronteras. Durante el seguimiento de la primera neoplasia es pertinente y urgente implementar y evaluar el impacto de programas de prevención primaria y secundaria de cáncer, sobre la incidencia de CM y la precocidad de su diagnóstico.

\section{AGRADECIMIENTOS}

Los autores agradecen la colaboración del personal del Registro de Tumores del Hospital Universitario Central de Asturias, el asesoramiento de Elena Belyakova, experta en bioestadística, y la financiación del Fondo de Investigación Sanitaria (FIS PI 03/0542) y de la "Obra Social Cajastur".

\section{BIBLIOGRAFÍA}

1. Working Group Report. International rules for multiple primary cancers (ICD-0 third edition). Eur J Cancer Prev. 2005; 14: 307-8.

2. Filali K, Hedelin G, Schaffer P, Esteve J, Arveux $\mathrm{P}$, Bouchardy $\mathrm{C}$, et al. Multiple primary cancers and estimation of the incidence rates and trends. Eur J Cancer. 1996; 32A: 683-90.

3. Sankila R, Pukkala E, Teppo L. Risk of subsequent malignant neoplasms among 470,000 cancer patients in Finland, 1953-1991. Int J Cancer. 1995; 60: 464-70.

4. Demandante CG, Troyer DA, Miles TP. Multiple primary malignant neoplasms: case report and a comprehensive review of the literature. Am J Clin Oncol. 2003; 26: 79-83.

5. Folgueras V, Astudillo A, Zaplana J. Registro de Tumores del Hospital Universitario Central de Asturias. Resumen del año 2002. Oviedo: Hospital Universitario Central de Asturias, Instituto Universitario del Principado de Asturias; 2007.

6. Travis LB, Rabkin CS, Brown LM, Allan JM, Alter BP, Ambrosone CB, et al. Cancer survivorship-genetic susceptibility and second primary can- 
cers: research strategies and recommendations. J Natl Cancer Inst. 2006; 98: 15-25.

7. Hemminki K, Aaltonen L, Li X. Subsequent primary malignancies after endometrial carcinoma and ovarian carcinoma. Cancer. 2003; 97: 2432-9.

8. Dong C, Hemminki K. Second primary neoplasms in 633,964 cancer patients in Sweden, 1958-1996. Int J Cancer. 2001; 93: 155-61.

9. Crocetti E, Buiatti E, Falini P, Italian Multiple Primary Cancer Working Group. Multiple primary cancer incidence in Italy. Eur J Cancer. 2001; 37: 2449-56.

10. Hayat MJ, Howlader N, Reichman ME, Edwards BK. Cancer statistics, trends, and multiple primary cancer analyses from the Surveillance, Epidemiology, and End Results (SEER) Program. Oncologist. 2007; 12: 20-37.

11. Heinävaara S, Sankila R, Storm H, Langmark F, Hakulinen T. Relative survival of patients with prostate cancer as a first or subsequent tumor - a Nordic collaborative study. Cancer Causes Control. 2002; 13: 797-806.

12. Doubrovsky A, Menzies SW. Enhanced survival in patients with multiple primary melanoma. Arch Dermatol. 2003; 139: 1013-8.

13. Goggins WB, Tsao H. A population-based analysis of risk factors for a second primary cutaneous melanoma among melanoma survivors. Cancer. 2003; 97: 639-43.

14. Bhattacharyya N, Nayak VK. Survival outcomes for second primary head and neck cancer: a matched analysis. Otolaryngol Head Neck Surg. 2005; 132: 63-8.

15. Izquierdo JN, Schoenbach VJ. The potential and limitations of data from population-based state cancer registries. Am J Public Health. 2000; 90: 695-98.

16. Hall S, Schulze K, Groome P, Mackillop W, Holowaty E. Using cancer registry data for survival studies: the example of the Ontario Cancer Registry. J Clin Epidemiol. 2006; 59: 67-76.

17. Anton-Culver H. Cancer prevention strategies: use of cancer prevention research registries. Environ Health Perspect. 1995; 103 (Suppl 8): 237-9.

18. Reves J, Ginard D, Vanrell M, Mariñó Z, Garau I, Llompart A, et al. Implantación y evaluación del cribado poblacional de la poliposis adenomatosa familiar. Med Clin (Bar). 2007, 129: 51-2.
19. Fritz A, Percy C, Jack A, Shanmugaratnam K, Sobin L, Parkin DM, et al. International Classification of Diseases for Oncology (ICD-O), Third Edition. Geneva: World Health Organization; 2001.

20. Parkin DM, Shanmugaratnam K, Sobin L, Ferlay J, Whelan SL. Histological Groups for comparative studies. IARC Technical Report No. 31. Lyon: IARC Press; 1998.

21. Boyle P, Autier P, Bartelink H, Baselga J, Boffetta $\mathrm{P}$, Burn $\mathrm{J}$, et al. European Code Against Cancer and scientific justification: third version (2003). Ann Oncol; 2003; 14: 973-1005.

22. Riffenburgh RH, Johnstone PA. Survival patterns of cancer patients. Cancer 2001; 91: 2469-75.

23. Artac M, Bozcuk H, Ozdogan M, Demiral AN, Sarper A, Samur M, et al. Different clinical features of primary and secondary tumors in patients with multiple malignancies. Tumori; 2005; 91: 317-20.

24. Roychoudhuri R, Evans H, Robinson D, Møller H. Radiation-induced malignancies following radiotherapy for breast cancer. Br J Cancer. 2004; 91: 868-72.

25. Páez A, Luján M, Llanes L, Romero I, de la Cal MA, Miravalles E, et al. PSA-use in a Spanish industrial area. Eur Urol. 2002; 41: 162-6.

26. Sant M, Aareleid T, Berrino F, Bielska Lasota M, Carli PM, Faivre J, et al. EUROCARE-3: survival of cancer patients diagnosed 1990-94--results and commentary. Ann Oncol. 2003; 14 Suppl: 61-118.

27. Sugimura T. Cancer prevention: past, present, future. Mutat Res. 1998; 402:7-14.

28. Demark-Wahnefried W, Aziz NM, Rowland JH, Pinto BM. Riding the crest of the teachable moment: promoting long-term health after the diagnosis of cancer. J Clin Oncol. 2005; 23: 581430 .

29. Rizzo M, Migneco A, Mansueto P, Tringali G, DI Lorenzo G, Rini GB. Therapeutic patient education in oncology: pedagogical notions for women's health and prevention. Eur J Cancer Care. 2007; 16: 9-11.

30. Giorgi R, Armanet A, Gouvernet J, Bonnier P, Fieschi M. Regression models for crude and relative survival: a comparative review. Rev Epidemiol Sante Publique. 2005 Sep; 53:409-17. 
31. Alvarez Marcos CA, Llorente Pendás JL, Franco Gutiérrez V, Hermsen MA, Franco Albalad MP, Fernández Espina H, et al. Segundos tumores primarios en el cáncer escamoso de cabeza y cuello. Acta Otorrinolaringol Esp. 2006; 57: 462-6.
32. Brenner H. Long-term survival rates of cancer patients achieved by the end of the $20^{\text {th }}$ century: a period analysis. Lancet. 2002; 360: 1131-5.

33. Weston R. Intervention and prevention. Eur J Cancer Prev. 2005; 8: 373-5. 